\title{
RELAÇÕES INTERORGANIZACIONAIS E COMPLEMENTARIDADE DE CONHECIMENTOS: PROPOSIÇÃO DE UM ESQUEMA CONCEITUAL
}

\section{INTER-ORGANIZATIONAL RELATIONSHIPS AND COMPLEMENTARITY} OF KNOWLEDGE: PROPOSAL OF A CONCEPTUAL FRAMEWORK

\author{
ALSONES BALESTRIN \\ Doutor em Administração pelo PPGA/UFRGS. \\ Professor e pesquisador do Programa de Pós-graduação da \\ Universidade do Vale do Rio dos Sinos (Unisinos). \\ Rua Coronel Feijó, 370, ap. 303 - Higienópolis - Porto Alegre - RS - CEP 90520-060 \\ E-mail: abalestrin@unisinos.br \\ JORGE RENATO VERSCHOORE \\ Doutor em Administração pela PPGA/UFRGS. \\ Professor e pesquisador da Universidade do Vale do Rio dos Sinos (Unisinos). \\ Avenida Unisinos, 950 - São Leopoldo - RS - CEP 93022-000 \\ E-mail: jorgevf@unisinos.br
}




\section{RESUMO}

O presente artigo aborda o tema sobre a complementaridade de conhecimentos entre Pequenas e Médias Empresas (PME) em um contexto em rede. Com base na revisão analítica das teorias de redes interorganizacionais, buscou-se a compreensão da relevância estratégica das redes na complementaridade de conhecimentos entre as empresas. Para aprofundar tal problemática, o artigo teve como objetivo geral a proposição de um esquema conceitual com a finalidade de auxiliar na compreensão da dinâmica de complementaridade de conhecimentos em um contexto das redes. A pesquisa empírica foi conduzida por meio de entrevistas com os dirigentes de empresas e instituições localizadas na Agivest (Brasil), na Tecnópole do Futuroscope (França) e no Pólo de Informática de São Leopoldo (Brasil). Os resultados da pesquisa sinalizam que a existência de um ambiente de sinergia e complementaridade de conhecimentos em um contexto vai muito além da proximidade física entre as empresas, sofrendo forte influência de elementos, como fatores contingências, coerência, conectividade, mecanismos de coordenação e espaços de interação.

\section{PALAVRAS-CHAVE}

Cooperação; Criação de conhecimento; Redes interorganizacionais; Pequenas e médias empresas (PME); Estratégia.

\section{ABSTRACT}

The present paper presents the dynamics of the complementarity knowledge between Small and Medium Firms (SMF) in a network context. Having as a starting point the analytical analysis of the inter-organizational, creation and knowledge management network theories, as well as, the innovation processes; it sought to understand the network strategies relevance, and, above all, the development of the SMF. Considering the theoretical evidences, the research matter faced to 
comprehend how the knowledge complementarity dynamics occur in a networking. In order to deepen this mater, it attempted, from the empirical evidences, to build a conceptual framework so as to assist the understanding of the functioning in network organized companies. The empirical research was carried out with interviews in managerial level from businesses and institutions based in Agivest (Brazil), in Futuroscope Tecnópole (France) and in Polo de Informática de São Leopoldo (Brazil). Summarizing, these results show that the existence of a synergy environment and knowledge complementarity in a network suffers the influence of elements as such: contingency pressures, coherence, connectivity, coordination mechanisms and interaction situations.

\section{KEYWORDS}

Cooperation; Knowledge creation; Inter-organizational network; Small and medium firms (SMF); Strategy.

\section{INTRODUÇÃO}

O tema das "redes interorganizacionais" tem tomado amplo espaço nos estudos organizacionais. Para Nohria e Eccles (I992), existem três razões principais para o aumento do interesse sobre o tema: a) a emergência da "nova competição", como a que está ocorrendo nos distritos industriais italianos e no Vale do Silício - se o "velho" modelo de organização era a grande firma hierárquica, o modelo da organização considerada característica da "nova competição" é a rede de inter-relações laterais intra e interfirmas; b) o surgimento das tecnologias de informação e comunicação (TIC) tem tornado possível uma maior capacidade de inter-relações entre firmas dispersas; e c) a consolidação da análise de redes como uma disciplina acadêmica não somente restrita a alguns grupos de sociólogos, mas expandida para uma ampla interdisciplinaridade dos estudos organizacionais.

Casos como os distritos industriais da Região da Emilia Romana também despertaram o interesse acadêmico para o tema. O elevado grau de associativismo e cooperação permite que as Pequenas e Médias Empresas (PME) do norte da Itália tenham competitividade internacional (CASAROTTO FILHO; PIRES, I999). Para Amato Neto (2000), o desenvolvimento de redes de empresas vem ganhando relevância não só para as economias de vários países industrializados, como Itália, Japão e Alemanha, mas também para os chamados países emergentes ou de economias em desenvolvimento, como é o caso do Brasil, do México, do Chile e da Argentina. Especificamente no Brasil, observam-se muitos exemplos de políticas públicas que buscam o estímulo à formação de arranjos produtivos 
locais, como os pólos industriais, as incubadoras de empresas, os parques industriais, as redes de cooperação, entre outros modelos. Essas tipologias de arranjos interorganizacionais visam criar um ambiente de sinergia, de cooperação, de complementaridade de conhecimentos em prol de um contexto propício à competitividade, sobretudo, no caso das PME (BALESTRIN; VARGAS, 2004).

A dinâmica de complementaridade de conhecimentos nesses "contextos em rede” (expressão utilizada neste artigo para referir-se aos diversos arranjos favoráveis às relações de cooperação interorganizacionais), no entanto, é resultado de um processo complexo, em que múltiplas dimensões poderão funcionar tanto para facilitar quanto para dificultar a interação entre os atores (empresas, instituições públicas, universidades, institutos de pesquisa etc.). O desafio de criação de um ambiente efetivo de sinergia e de inovação nesses contextos levou à seguinte questão para a presente pesquisa: Quais os elementos críticos na dinâmica de complementaridade de conhecimentos entre as empresas em um contexto em rede? Buscar-se-á, por meio de evidências teóricas e empíricas, aprofundar tal questionamento, com o objetivo central de propor um esquema conceitual em que serão apresentados os elementos críticos presentes na dinâmica de complementaridade de conhecimentos nos contextos em rede. Entende-se por complementaridade de conhecimentos a troca ou partilha de informações, conhecimentos, experiências, know-how e habilidades, por meio de processos colaborativos, entre empresas e demais atores.

Para alcançar o objetivo proposto, o artigo está estruturado da seguinte forma: inicia-se com uma reflexão conceitual sobre a dinâmica de complementaridade de conhecimentos em um contexto em rede; logo após, serão descritos os aspectos metodológicos da pesquisa; na seqüência, serão apresentadas as implicações teóricas dos resultados empíricos, bem como o esquema conceitual. Ao final, serão destacadas algumas recomendações para os casos pesquisados, indicadas as limitações da pesquisa e sugeridas linhas para pesquisas futuras.

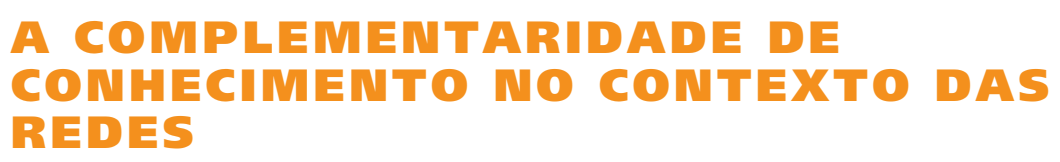

A reflexão teórica para o presente estudo teve a sua origem na análise de algumas contribuições envolvendo a temática de redes interorganizacionais. Entre elas, podem-se destacar: o "estado da arte” sobre redes (OLIVER; EBERS, I998); as tipologias de redes (MARCON; MOINET, 2000); a viabilidade econômica e estratégica das redes (JARILLO, I988; EBERS; JARILLO, I998) e as tipologias de redes de PME (PERROW, I992; HUMAN; PROVAN, I997). Outras pesqui- 
sas recentes (POWELL, I998; NONAKA; NISHIGUCHI, 200I; TSAI, 2002; SPENCER, 2003; FLORÉN; TELL, 2004; RODAN; GALUNIC, 2004) indicam a importância das relações interorganizacionais para o processo de complementaridade de conhecimento, sinalizando que uma rede de empresas poderá ser mais eficaz que uma firma "isolada" no processo de criação, de transferência e de recombinação de conhecimentos.

Tal atributo das redes torna-se relevante ao considerar a afirmação de Kogut e Zander (I992) e Grant (I996) de que um processo efetivo de criação de novos conhecimentos poderá torna-se uma importante fonte de sustentabilidade de vantagens competitivas para as empresas. Dessa forma, as redes interorganizacionais tornam-se opções estratégicas a serem consideradas ante os novos desafios de competitividade das organizações, sobretudo por fortalecer os processos de inovação (MOWERY; ROSENBERG, I989; ROTHWELL, I995).

A revisão analítica dessas contribuições permitiu a compreensão da relevância estratégica das redes em ambientes competitivos, especialmente pelo fato de possibilitar a sinergia de esforços entre empresas em direção à complementaridade de conhecimentos úteis para os processos organizacionais. No entanto, a maior parte das pesquisas desenvolvidas tangenciou a problemática de como ocorre a dinâmica de complementaridade de conhecimentos no contexto das redes e quais os elementos críticos implicados nesse processo. Algumas teorizações evidenciaram elementos críticos presentes na formação e no funcionamento das redes, como no caso dos seguintes trabalhos: Oliver (I990), ao demonstrar a influência de pressões contingenciais na formação e no funcionamento das empresas em rede; Castells (I999), ao argumentar que o desempenho de uma determinada rede dependerá de dois de seus atributos fundamentais - conectividade e coerência; e Grandori e Soda (I995), ao destacarem a importância dos mecanismos de coordenação na governança dos atores em uma rede. Na sequência deste item, buscam-se aprofundar essas dimensões no sentido de melhor compreender o funcionamento das redes e suas possíveis relações com a dinâmica de complementaridade de conhecimentos.

\subsection{PRESSÕES CONTINGENCIAIS}

Uma das questões relevantes nos estudos sobre redes é entender as pressões ou motivações que levam à formação das redes interorganizacionais. Para Oliver (I990), as redes são formadas a partir de pressões contingenciais que forçam as empresas a trabalhar de forma conjunta, apresentando seis contingências determinantes: a) Necessidade - uma organização freqüentemente estabelece elos ou trocas com outras organizações por necessidade. Essa contingência está sustentada por estudos oriundos sobretudo das abordagens de dependência de 
recursos; b) Assimetria - sob essa contingência, as relações interorganizacionais são induzidas pelo potencial exercício de poder de uma organização sobre outra; c) Reciprocidade - de acordo com essa perspectiva, as redes interorganizacionais ocorrem com o propósito de buscar interesses e objetivos comuns em ações de cooperação; d) Eficiência - a eficiência é a única dessas seis contingências que apresenta uma orientação interna ao buscar uma melhor performance na eficiência organizacional que as redes são estabelecidas; e) Estabilidade - a incerteza induz as organizações a estabelecer e gerenciar inter-relações para encontrar estabilidade no ambiente; f) Legitimidade - é sustentada fundamentalmente pela teoria institucional, a qual sugere que o ambiente institucional impõe pressões sobre as organizações para justificar suas atividades e seus resultados.

Oliver (I990) argumenta que essas contingências são a causa que induz ou motiva as organizações a estabelecer relações interorganizacionais, isto é, elas explicam as razões pelas quais as organizações escolhem relacionar-se com outras. Embora cada determinante seja uma causa suficientemente separada para a formação de tais relacionamentos, essas contingências, em geral, ocorrem simultaneamente. Dessa maneira, segundo a análise das pressões contingenciais, subjacentes à formação das redes interorganizacionais poderão estar as seguintes razões: exercer influência sobre reguladores (assimetria); promover a coletividade entre os membros mediante o compartilhamento de informações (reciprocidade); obter vantagem econômica, como melhores recursos e fornecedores (eficiência); reduzir a incerteza competitiva por meio de esforços para padronizar produtos ou serviços de cada ator da rede (estabilidade) e melhorar a imagem da rede e de seus atores (legitimidade).

\subsection{CONECTIVIDADE ENTRE OS ATORES}

Segundo a análise de Castells (I999), a robustez de uma rede está em função do grau de conectividade, ou seja, de comunicação entre um ator e outro. Logo, por definição, não existirá rede caso os elos não se comuniquem. A conectividade poderá ser possibilitada tanto pelos recursos de comunicação (aparatos tecnológicos ou mecânicos que transmitem, armazenam e processam dados) quanto pelo contato face a face. Para Caglio (I998), como conseqüência do potencial de uso dos recursos de TIC, o fluxo de informação tem-se tornado hoje a principal variável explicativa do processo que leva à formação e ao funcionamento de redes interorganizacionais.

Verifica-se, então, que uma das principais conseqüências das "modernas" TIC (como a internet) foi facilitar a comunicação de informações e conhecimentos entre a empresa e outros atores presentes em seu ambiente institucional. A premissa básica é que as TIC melhoram a disseminação da informação, criam e 
suportam formas complexas de comunicação e interação, proporcionando condições para a inovação (POWELL, I990). No entanto, os efeitos de comunicação das TIC devem ser relativizados, pois nem todas as redes interorganizacionais estão preparadas (em seus recursos humanos e físicos) para potencializar todos esses recursos (NOHRIA; ECCLES, I992). Da mesma forma, parte considerável dos conhecimentos presentes em uma rede são tácitos, o que dificulta um suporte total pelos recursos das TIC.

Para existir elevada conectividade, Hage e Hollingsworth (2000) ressaltam que deverá ocorrer uma freqüente e intensa comunicação face a face entre os vários atores de uma rede interorganizacional. Quanto mais freqüente e intensa for essa comunicação, maior será a quantidade de conhecimento tácito comunicado. Talvez seja essa uma das explicações do sucesso dos distritos industriais do norte da Itália, pois, conforme Brown e Hendry (I998), existe uma densa circulação de pessoas entre as firmas, o que fomenta a troca direta de conhecimentos. Salienta-se que a conectividade em uma rede poderá utilizar-se dos benefícios das TIC; no entanto, a interação face a face deve ser preservada, sobretudo para a comunicação de conhecimentos tácitos ou desestruturados.

\subsection{NÍVEIS DE COERÊNCIA}

O desempenho de uma determinada rede está relacionado ao aspecto da coerência, isto é, na medida em que há interesses compartilhados entre os objetivos da rede com os objetivos de seus atores (CASTELLS, I999). Para que a coerência exista, é necessário um ambiente que possibilite que a cooperação seja no mínimo superior à competição entre as empresas participantes do ambiente da rede. Segundo Gambetta (2000), para ocorrer cooperação, deverá existir certo nível de confiança mútua. Caso a desconfiança seja completa ou unilateral, a cooperação certamente falhará. Jarillo (I988), em seu estudo sobre estratégia em rede, demonstrou a importância da confiança nas inter-relações como fator que reduz os custos de transação e torna a existência das redes economicamente viáveis. Para Boss (I978), em uma "atmosfera" de confiança os problemas são resolvidos mais eficientemente, porque a informação e o know-how são trocados mais livremente.

O nível de coerência também poderá ser influenciado pelas pressões contingenciais que levaram à formação de uma rede. Dessa maneira, uma rede formada sob a contingência de reciprocidade e de necessidade provavelmente terá uma tendência maior para a cooperação em relação à rede formada sob a contingência da legitimidade. Outra dimensão que poderá ter um papel relevante nos níveis de coerência são os mecanismos de coordenação implicados na governança das relações entre os atores. 


\subsection{MECANISMOS DE COORDENAÇÃO}

Entendem-se como mecanismos de coordenação as formas de governança entre os interesses da rede e os interesses de seus atores, por meio de uma variedade de instrumentos que tentam governar o comportamento, estruturando informações relevantes e estabelecendo regras de conduta (CAGLIO, I998). Para melhor entenderem o papel da coordenação nas redes, Grandori e Soda (I995) compilaram uma série de mecanismos de coordenação (controle e coordenação social, relações de autoridade e hierarquia, sistema de controle e planejamento, sistemas de incentivos, sistemas de seleção, suporte público, negociação e comunicação e tecnologias de informação e de comunicação) utilizados por diferentes tipologias de redes interorganizacionais. Esses diferentes mecanismos poderão ser utilizados em vários graus de combinações no processo de coordenação de uma rede. No entanto, Casson e Cox (1997) salientam a importância do fluxo de informação na coordenação do fluxo de atividades e recursos da rede. Segundo Miles e Snow (i986), para que as redes possam funcionar adequadamente, é necessário um processo de comunicação eficiente entre os atores. A argumentação desses autores indica que em uma rede na qual a informação não circula adequadamente ocorrerão dificuldades para estabelecer objetivos e interesses comuns e outras atividades que levarão à cooperação.

Esses quatro elementos conceituais (pressões contingenciais, coerência, conectividade e mecanismos de coordenação) fornecem indícios relevantes de como as redes são formadas e como ocorre o seu funcionamento. Pela importância e consistência de tais evidências, apresenta-se como proposição teórica inicial, para a verificação empírica no campo de pesquisa, de que tais elementos também poderão estar relacionados com a dinâmica de complementaridade de conhecimentos. Os resultados da pesquisa deverão indicar a pertinência desses elementos, bem como identificar outros elementos críticos que influenciam esse processo.

\section{METODOLOGIA DA PESQUISA}

A pesquisa foi realizada por meio do estudo de múltiplos casos (unidades de análise) selecionados com base na conveniência e no alinhamento com o foco da pesquisa. Apresenta-se a seguir uma breve descrição de cada um dos casos pesquisados:

a) Associação Gaúcha da Indústria do Vestuário (Agivest) - A Agivest é formada por 35 pequenas indústrias do setor têxtil e do segmento de confecções, loca- 
lizada na região de Ijuí, Rio Grande do Sul (Brasil). Sua constituição ocorreu em setembro de 200I com os seguintes objetivos: ampliação de mercado, melhorias tecnológicas e alcance de maior competitividade para as pequenas empresas participantes por meio do fortalecimento da cooperação. A escolha dessa unidade de análise é justificada: por ser uma rede formada de PME que estabelecem relações horizontais para alcançarem desenvolvimento coletivo; por ser uma rede de PME industriais que perseguem a inovação em seus produtos para melhor competirem em mercados nacionais.

b) Tecnópole do Futuroscope - A Tecnópole do Futuroscope foi criada há vinte anos na região de Poitiers (França) com o objetivo de desenvolver uma região identificada pela economia agroalimentar e pelo setor terciário mais voltado à administração pública. Diferentemente de outras Tecnópoles, o projeto da Tecnópole do Futuroscope apresenta uma proposta original ao estar ancorado em três pilares - lazer, negócio e pesquisa -, e estar localizado em um espaço geográfico de oitenta hectares. Atualmente o projeto está consolidado, acolhendo mais de cem PME do segmento de TIC, uma estrutura de hotéis e serviços, o primeiro parque da imagem da Europa que atrai aproximadamente 2,5 milhões de turistas ao ano e uma estrutura de pesquisa que conta com aproximadamente setecentos pesquisadores e dois mil estudantes. A escolha dessa unidade de análise foi motivada pelos seguintes fatos: reúne um grupo de empresas da chamada "nova economia”, intensivas em conhecimento e inovação e pelo projeto inovador da Tecnópole do Futuroscope que poderá apresentar uma dinâmica de funcionamento diferente dos casos estudados no Brasil e podendo proporcionar importantes elementos de análise.

c) Pólo de Informática - O Pólo de Informática foi criado em 30 de junho de I999 na cidade de São Leopoldo, região metropolitana de Porto Alegre (Brasil). Projetado para acolher empresas de base tecnológica e gerar um ambiente de sinergia entre empresas, universidade e demais atores, sua estrutura engloba uma incubadora empresarial, um condomínio de empresas e um parque tecnológico. Atualmente, existem 22 empresas residentes no Pólo, e na incubadora e no condomínio, ambos mantidos pela Universidade do Vale do Rio dos Sinos (Unisinos), existem doze empresas, enquanto no parque tecnológico existem dez empresas. A escolha dessa unidade de análise é justificada por: reunir um grupo de empresas de base tecnológica; haver uma Universidade como forte articuladora do processo; constituir um arranjo de empresas um tanto diverso da Agivest e da Tecnópole, possibilitando, assim, uma melhor compreensão teórica do funcionamento de empresas em um contexto em rede. 
Deve-se salientar que os casos escolhidos apresentam pontos similares e divergentes, conhecidos a priori do estudo de campo. As semelhanças estão nas seguintes características: a) os três casos são resultados de políticas públicas para o desenvolvimento regional e empresarial; b) todos os casos apresentam o mesmo propósito - criar as condições necessárias para um ambiente de cooperação, sinergia e inovação; c) todos os casos são formados majoritariamente por PME; d) nos três casos existem algumas condições que poderão facilitar a interação entre as empresas, como a proximidade geográfica e alguns interesses comuns.

Os pontos divergentes encontram-se nas seguintes características: a) enquanto as empresas da Tecnópole do Futuroscope e do Pólo de Informática estão concentradas em um mesmo local geográfico, a Agivest está mais dispersa, abrangendo empresas em um raio de oitenta quilômetros; b) a Agivest é formal e juridicamente constituída e gerenciada por meio de mecanismos legais (assembléia, código de ética, regimento interno e estatuto), enquanto a Tecnópole do Futuroscope e o Pólo de Informática apresentam uma estrutura de coordenação mais informal; c) enquanto no Pólo de Informática e na Tecnópole as empresas apresentam elevado nível de desenvolvimento tecnológico, na Agivest observa-se o inverso. Para a análise dos casos, adotou-se uma abordagem complementar de evidências, em vez de propriamente uma replicação comparativa de casos. Apesar da diversidade de evidências que os diferentes casos poderão apresentar, o que demanda um maior cuidado e complexidade de análise, acredita-se que eles cobrirão de maneira mais satisfatória a heterogeneidade e a variabilidade dos elementos conceituais envolvidos na pesquisa.

A operacionalização do estudo de campo ocorreu por meio da sistematização entre os elementos conceituais de análise (identificados na revisão da literatura) e as variáveis correspondentes, conforme o Quadro I. Essa lógica de estruturação permitiu uma melhor adequação entre as variáveis a serem observadas e as questões da entrevista semi-estruturada. 
QUADRO I

OPERACIONALIZAÇÃO DAS VARIÁVEIS DA PESQUISA

\begin{tabular}{|c|c|}
\hline ELEMENTOS CONCEITUAIS & VARIÁVEIS DA PESQUISA \\
\hline $\begin{array}{l}\text { Pressões } \\
\text { Contingenciais }\end{array}$ & Motivações e objetivos que levaram à empresa a participar da rede \\
\hline Conectividade & $\begin{array}{l}\text { Nível de interação entre as empresas; Formas de comunicação na } \\
\text { rede; Nível de utilização de TIC }\end{array}$ \\
\hline Coerência & $\begin{array}{l}\text { Nível de cooperação e de competição entre as empresas na rede; } \\
\text { Contratos ou projetos de cooperação entre as empresas da rede; } \\
\text { Interesses comuns partilhados com outras empresas da rede }\end{array}$ \\
\hline $\begin{array}{l}\text { Mecanismos de } \\
\text { coordenação }\end{array}$ & $\begin{array}{l}\text { Formas de coordenação da rede; Instrumentos de coordenação } \\
\text { utilizados; Responsabilidade pela coordenação }\end{array}$ \\
\hline $\begin{array}{l}\text { Complementaridade } \\
\text { de conhecimentos }\end{array}$ & $\begin{array}{l}\text { Quais os conhecimentos comunicados entre as empresas da rede e } \\
\text { quais as contribuições para a empresa }\end{array}$ \\
\hline $\begin{array}{l}\text { Características gerais } \\
\text { dos casos }\end{array}$ & $\begin{array}{l}\text { Número de empregados das empresas; Idade da empresa; Tempo } \\
\text { em que a empresa está instalada na rede; Segmento de negócio } \\
\text { da empresa; Tipo de produto ou serviço da empresa; Nível de } \\
\text { desenvolvimento tecnológico da empresa }\end{array}$ \\
\hline $\begin{array}{l}\text { Outros elementos } \\
\text { críticos }\end{array}$ & $\begin{array}{l}\text { Outros elementos que poderão influenciar a complementaridade } \\
\text { de conhecimentos no contexto de uma rede }\end{array}$ \\
\hline
\end{tabular}

O pesquisador utilizou um roteiro de entrevista semi-estruturado (elaborado com base nas variáveis de pesquisa constantes no Quadro i) com o objetivo de apresentar uma seqüência lógica das questões aos entrevistados, que foi conduzido da seguinte forma: a) Tecnópole do Futuroscope - foram realizadas treze entrevistas com dirigentes de empresas selecionadas aleatoriamente de um total de cem empresas do segmento de TIC; b) Agivest - a operacionalização dessa fase ocorreu por meio de entrevistas realizadas com o presidente da rede e com quatro dirigentes de PME pertencentes à Agivest, selecionados aleatoriamente de um total de 35 empresas; c) Pólo de Informática de São Leopoldo - foram realizadas cinco entrevistas: com o diretor da Unitec (incubadora do Pólo de Informática mantida pela Unisinos), com dois dirigentes de empresas localizadas no parque tecnológico e com dirigentes de duas empresas localizadas na incubadora. Os dirigentes entrevistados foram selecionados aleatoriamente de um total de 22 empresas. 
As entrevistas tiveram uma duração média de 40 minutos e ocorreram nos anos de 2004 e 2005. Deve-se destacar que a definição do número de entrevistas não teve nenhuma relação com a representatividade estatística, mas sim buscar um nível de evidências empíricas suficientes ante a problemática de pesquisa. Diante disso, o número de entrevistas na Tecnópole teve de ser maior em razão da dificuldade de pesquisa proporcionada pelo contexto cultural não familiar para o pesquisador. Para a interpretação dos resultados, as entrevistas foram transcritas e, posteriormente, os relatórios foram submetidos a análise de conteúdo. Os três casos foram analisados em um primeiro momento individualmente e depois em conjunto, procurando-se identificar os elementos semelhantes e divergentes que poderiam refletir-se nas implicações da pesquisa.

\section{RESULTADOS DA PESQUISA E IMPLICAÇÕES TEÓRICAS DECORRENTES}

Os resultados da pesquisa demonstraram uma diversidade ampla no nível de complementaridade de conhecimentos entre os três casos pesquisados, apresentando níveis elevado, baixo e médio, respectivamente, na Agivest, na Tecnópole e no Pólo de Informática (Quadro 2). No caso da Agivest, a dinâmica de complementaridade possibilitou conhecimentos, sobretudo, de mercado (fornecedores, representantes, canais de distribuição, tendências, matérias-primas) e de processos e tecnologias de produção. Essa dinâmica de interação e complementaridade de conhecimentos entre as empresas da Agivest possibilitou ganhos para as empresas associadas, especialmente em melhorias e inovações incrementais de produtos e de processos.

QuADro 2

COMPLEMENTARIDADE DE CONHECIMENTOS

AGIVEST

Conhecimentos sobre:

fornecedores, representantes, processos e tecnologias de produção, canais de distribuição, matérias-primas, tendências de mercado, técnicas de gestão e oportunidades de vendas

\section{TECNÓPOLE DO FUTUROSCOPE PÓLO DE INFORMÁTICA}

Conhecimentos sobre: Conhecimentos sobre: desenvolvimento de novas tecnologias, produtos e técnicas de conceitos e técnicas de produção produção, novos produtos e oportunidades de vendas 
No Pólo de Informática, alguns empresários buscaram conhecimentos sobre novas tecnologias junto a colegas e outros profissionais de empresas localizadas no Pólo. Para outros empresários, a possibilidade de ter acesso a conhecimentos científicos e de desenvolver projetos com a Unisinos é um forte diferencial ante os concorrentes localizados fora do contexto do Pólo. Já na Tecnópole, a dinâmica de complementaridade de conhecimentos foi bem menos ativa, existindo somente alguns casos de cooperação em conhecimentos técnicos entre as empresas, como em projetos para desenvolvimento de produtos.

Os resultados demonstram que, enquanto na Agivest a ação coletiva em rede está trazendo resultados positivos na complementaridade de conhecimentos e na competitividade das PME, na Tecnópole do Futuroscope a cooperação entre as empresas foi bem menos intensa. Tais evidências instigam alguns questionamentos: Por qual motivo na Tecnópole a complementaridade de conhecimentos está aquém do esperado? Quais são os fatores que promovem uma elevada sinergia entre as empresas da Agivest? No caso do Pólo de Informática, quais os elementos explicativos de um nível de complementaridade maior em relação à Tecnópole, porém menor em relação à Agivest? A resposta a esses questionamentos, alinhados aos elementos conceituais (pressões contingenciais, conectividade, coerência e mecanismos de coordenação) evidenciados na revisão teórica, será o foco analítico a ser explorado na seqüência da análise dos resultados.

\section{RELAÇÃO DAS PRESSÕES CONTINGENCIAIS COM A COMPLEMENTARIDADE DE CONHECIMENTOS}

Conforme resultados apresentados na Figura I, observa-se que na Agivest a necessidade tem sido uma das contingências de primeira ordem na motivação das empresas ao participarem de uma configuração em rede. O baixo nível de desenvolvimento tecnológico das indústrias de confecções e um segmento de alta competição, provocado pela concorrência com outros pólos industriais localizados em Santa Catarina e São Paulo, tornaram vulnerável a sobrevivência dessas PME. A possibilidade de reciprocidade (cooperação e colaboração) surgiu como a segunda variável contingencial que pressionou as PME a engajarem-se no projeto Agivest. Algumas teorias na área de estratégia e custos de transação (JARILLO, I988) indicam que a reciprocidade com outras empresas poderá ser uma eficiente escolha estratégica de competitividade, sobretudo por parte das PME que encontram cada vez mais dificuldade de competir individualmente. As evidências do caso da Agivest apontam para as variáveis contingenciais de necessidade e de reciprocidade como as principais pressões que motivaram as empresas a participar de uma configuração em rede. 
As variáveis contingenciais de reciprocidade e de legitimidade foram as principais pressões que levaram as empresas a participar do Pólo de Informática. A possibilidade de desenvolver projetos conjuntos entre as empresas e com a Unisinos tem sido um dos principais fatores que levaram à instalação das PME no Pólo. O fato é que as empresas estão em um segmento com uma demanda por produtos crescentemente complexos em termos de competências e de conhecimentos, e em muitos casos as PME necessitam trabalhar em conjunto com outras empresas ou com a Universidade para desenvolvê-los. A legitimidade foi a segunda principal motivação que levou as PME a participar do Pólo. A proximidade com a Unisinos e a participação em um pólo de TIC promovem uma imagem positiva para as PME no mercado. O nível médio de complementaridade de conhecimentos encontrado no Pólo poderá estar associado a essas duas variáveis contingenciais. Se, por um lado, a complementaridade de conhecimentos é facilitada pelas empresas motivadas pela reciprocidade; por outro, ela nem sempre é buscada pelas empresas que visam somente legitimar-se no mercado perante a imagem.

A variável contingencial de legitimidade foi a que teve maior impacto na motivação das empresas em se estabelecer na Tecnópole. O conceito de um pólo de "novas tecnologias" tem proporcionado uma imagem positiva para as PME lá instaladas. A preocupação com a imagem pode estar relacionada a certo preconceito com as empresas localizadas fora da região parisiense. A explicação a esse fenômeno já foi trabalhada pela teoria institucional, como nos trabalhos de DiMaggio e Powell (1983), demonstrando que as empresas procuram fazer parte de uma "rede" para evitar o isolamento e obter legitimidade em seu ambiente institucional. Dessa forma, as PME terão uma imagem positiva no mercado ao fazerem parte de uma tecnópole associada às TIC, em relação às PME localizadas fora desse contexto.

Uma segunda motivação para as empresas instalarem-se na Tecnópole é a possibilidade de maior eficiência, resultado da infra-estrutura física e dos serviços disponíveis, e a possibilidade de redução dos custos de operação. A relação dessas variáveis contingenciais com o baixo nível de complementaridade de conhecimentos encontrado na Tecnópole pode ser explicada pelos seguintes fatores: para atender às pressões contingenciais de legitimidade e de eficiência não é necessária a existência de cooperação com outras empresas; alguns empresários manifestaram que suas empresas são altamente especializadas em certos domínios de TIC e que dificilmente encontrariam algum tipo de complementaridade com outras empresas que justificassem projetos de cooperação; as empresas apresentam um elevado nível de desenvolvimento tecnológico e recebem fortes incentivos públicos, logo a necessidade de cooperação não tem sido ainda percebida como uma opção estratégica de sobrevivência para tais empresas; as PME dominam conhe- 
cimentos estratégicos em determinadas esferas de tecnologia, o que promove certo temor em partilhar tais conhecimentos com outras empresas.

As implicações teóricas dos resultados encontrados sinalizam que a dinâmica de complementaridade de conhecimentos pode ser explicada, em parte, pelas variáveis contingenciais que pressionam as empresas a participar de um contexto em rede. Diante disso, identificou-se que o nível de cooperação e complementaridade de conhecimentos tem como forte fator influenciador a pressão contingencial de necessidade, que ocorre sobretudo pela hostilidade ambiental, como no caso da Agivest. Essa evidência está de acordo com os resultados da pesquisa de Villwock (200I) ao concluir que, em muitos contextos, a cooperação entre empresas não é estabelecida pelo fato de a hostilidade ambiental não ser tão intensa.

\section{FIGURA I}

VARIÁVEIS CONTINGENCIAIS E NÍVEIS DE COMPLEMENTARIDADE DE CONHECIMENTOS

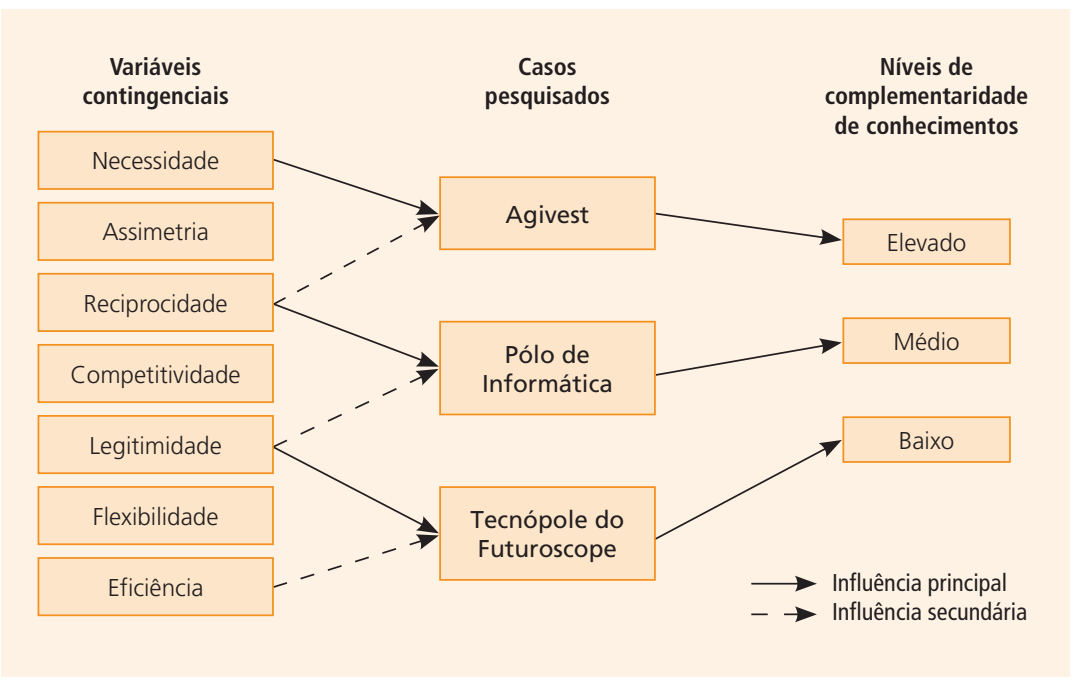

As evidências teóricas e os resultados empíricos demonstram que as teorizações de Oliver (I990) sobre o efeito das pressões contingenciais sobre o funcionamento das empresas em rede também se aplicam à dinâmica de complementaridade de conhecimentos, conduzindo à primeira proposição do esquema conceitual:

Proposição I - Diferentes variáveis contingenciais que pressionam as empresas a ingressar em uma rede estarão relacionadas à dinâmica de complementaridade de conhecimentos. 


\subsection{RELAÇÃO DO NÍVEL DE CONECTIVIDADE COM A COMPLEMENTARIDADE DE CONHECIMENTOS}

As evidências empíricas demonstraram níveis diferenciados de conectividade entre os atores nos casos pesquisados, apresentando um nível elevado na Agivest, um nível médio no Pólo de Informática e um nível baixo na Tecnópole do Futuroscope. Na Agivest, a elevada conectividade tem sido proporcionada pelas relações informais face a face entre os empresários. Essas evidências estão alinhadas aos trabalhos de Hage e Hollingsworth (2000) ao ressaltarem que a intensa comunicação face a face entre os vários atores em uma rede interorganizacional tem um papel fundamental no nível de conectividade. A eficácia da troca direta de conhecimentos entre os empresários, conforme já observado por Brown e Hendry (I998) nos distritos industriais do norte da Itália, também pode ser um dos fatores relevantes para explicar o elevado nível de complementaridade de conhecimentos que ocorre entre as empresas da Agivest.

Nos casos da Tecnópole e do Pólo de Informática, os níveis inferiores de conectividade em relação à Agivest tornam-se um elemento de influência negativa na dinâmica de complementaridade de conhecimentos entre as PME. Cabe salientar que, mesmo que as empresas da Tecnópole e do Pólo tenham um uso intensivo de TIC, internamente, isso não está refletindo no suporte ou na promoção de um nível de conectividade superior aos encontrados na Agivest. Esses resultados podem ser ratificados pela tese de Symon (2000) ao chamar de "falácia" a idéia de que o simples aumento dos links de comunicação eletrônica em uma rede possa superar as barreiras de comunicação e de participação dos atores. Alinhados a esses resultados, pode-se destacar Ciborra e Patriotta (I996), ao enfatizarem que as TIC não podem criar per se um maior ambiente de participação, sugerindo que a emergência de interação no contexto de uma rede depende muito mais da preexistência de condições culturais e contextuais facilitadoras.

À luz do pressuposto de Castells (I999) de que, por definição, não existirá rede caso os elos não se comuniquem, pôde-se observar que no caso da dinâmica de complementaridade de conhecimentos tal premissa se aplica. Logo, as implicações decorrentes dessas evidências demonstram que o nível de complementaridade de conhecimentos em um contexto terá forte influência do nível de interação e comunicação entre os seus atores. Essas evidências conduzem à segunda proposição para o esquema conceitual:

Proposição 2 - O nível de conectividade em um contexto em rede influencia a dinâmica de complementaridade de conhecimentos entre os seus atores. 


\subsection{RELAÇÃO DO NÍVEL DE COERÊNCIA COM A COMPLEMENTARIDADE DE CONHECIMENTOS}

Conforme os resultados do Quadro 3, observa-se que no contexto da Tecnópole, existe uma baixa coerência entre os interesses dos atores lá presentes. Ou seja, existem poucos objetivos comuns capazes de mobilizar a ação cooperada de um grupo de empresas e criar um efeito de sinergia. Na Agivest, a existência de vários projetos com objetivos comuns, como venda e compra em conjunto, marca única, entre outros, tem mobilizado as empresas em atividades de cooperação e proporcionado um contexto de maior complementaridade de conhecimentos.

QUADRO 3

OBJETIVOS COMUNS

AGIVEST

Utilização de marca comum

Compra conjunta de suprimentos

Venda conjunta de produtos

Lobbying

Ações de marketing
TECNÓPOLE DO

FUTUROSCOPE
PÓLO DE INFORMÁTICA

Na Tecnópole, muitos dos entrevistados afirmaram que um dos problemas da falta de dinâmica e sinergia é a inexistência de objetivos comuns que possam despertar o interesse pelo engajamento e pela cooperação entre as empresas. Tal problemática também ocorre entre as empresas do Pólo de Informática, conforme já evidenciado em outra pesquisa (WOLFFENBÜTTEL, 200I). Na Agivest, mesmo sendo formado por empresas dispersas em um espaço geográfico de aproximadamente $80 \mathrm{~km}$, ocorreu uma maior interação em relação aos outros dois casos em que as empresas estão fisicamente mais próximas. Esses dados apontam que a dinâmica de complementaridade e cooperação em um ambiente em rede é resultado muito mais da existência de projetos com interesses comuns e por uma eficiente coordenação, do que propriamente pela suntuosidade da estrutura disponível ou pela proximidade física do atores. Tais evidências empíricas conduzem à terceira proposição para o esquema conceitual:

Proposição 3 - A coerência, ou seja, a existência de objetivos comuns compartilhados em um contexto em rede influencia a dinâmica de complementaridade de conhecimentos entre as empresas. 


\subsection{RELAÇÃO DOS MECANISMOS DE COORDENAÇÃO COM A COMPLEMENTARIDADE DE CONHECIMENTOS}

Observou-se que na Agivest (Quadro 4) a assembléia constitui-se em um relevante mecanismo de coordenação, possibilitando que os interesses da maioria dos empresários sejam preservados. Na Tecnópole, a coordenação fica a cargo do CG (Conseil Général de la Vienne), que é o órgão público responsável por todas as decisões que envolvem os interesses da Tecnópole e de suas empresas. Já no Pólo de Informática, a coordenação fica um tanto ambígua, e os interesses da incubadora ficam a cargo da Unitec, e as decisões mais amplas que abrangem o Pólo ficam a cargo de um conselho geral, formado por um representante de cada uma das entidades participantes (Unisinos, Prefeitura Municipal, Associação Industrial e Comercial, entre outros).

\section{QUADRO 4}

\section{MECANISMOS DE COORDENAC ÃO}

\section{AGIVEST}

Presidência e conselho fiscal

Assembléia geral

Estatuto e regimento interno

Código de ética

Planejamento estratégico

Seleção das empresas
TECNÓPOLE DO FUTUROSCOPE

PÓLO DE INFORMÁTICA

Tanto na Tecnópole quanto no Pólo de Informática, os mecanismos de coordenação são utilizados de maneira menos intensa e mais hierárquica, respectivamente, em ambos os casos pelo CG e pela Unitec. Algumas das evidências empíricas demonstraram que a falta de uma articulação ou de uma "animação" mais efetiva tem contribuído para a falta de sinergia e de iniciativas de cooperação entre essas empresas. Já no caso da Agivest, a utilização de certos mecanismos de coordenação (como a assembléia, o estatuto, o código de ética, o regimento interno etc.) tem facilitado ações de cooperação e promovido melhor nível de coerência entre as empresas.

As implicações dos resultados ratificam o argumento de Grandori e Soda (I995), ao afirmarem que os mecanismos de coordenação influenciam no funcionamento de uma rede por garantir a governança e restringir a ação oportunista. A coordenação atua diretamente nos níveis de coerência da rede, promovendo a manutenção dos interesses coletivos aos interesses individuais. Essas evidências implicam a quarta proposição para o esquema conceitual: 
Proposição 4 - Os mecanismos de coordenação influenciam diretamente no nível de coerência e indiretamente no nível de complementaridade de conhecimentos entre as empresas de uma rede.

\subsection{RELAÇÃO DE OUTROS ELEMENTOS IDENTIFICADOS COM A COMPLEMENTARIDADE DE CONHECIMENTO}

Uma das características observada nos três casos pesquisados refere-se aos "espaços de interação" (expressão utilizada neste artigo para denominar os espaços ou momentos que permitem a comunicação face a face entre os atores em uma rede) capazes de criar um contexto favorável a complementaridade de conhecimento entre os empresários. Esse fato chamou a atenção do pesquisador e, provavelmente, foi a evidência mais original da pesquisa - a existência de espaços de interação que permitam a comunicação informal e face a face está fortemente relacionada com o nível de complementaridade de conhecimentos. Tais evidências podem complementar as teorizações de Suchman (I987) ao argumentar que o conhecimento não existe somente na cognição dos indivíduos, necessitando de um contexto específico em termos de tempo, de espaço e de relacionamento entre esses indivíduos para que possa ser capitalizado e expandido. Os resultados da pesquisa, conforme o Quadro 5, indicam que, na Agivest, a existência de espaços de interação mais informais (visitas e reuniões nas indústrias; viagens e visitas a feiras; confraternizações; reuniões informais etc.) que possibilitam aos empresários socializar suas habilidades, suas experiências e seu know-how, por meio da comunicação face a face, teve um papel relevante na conectividade e na complementaridade de conhecimentos.

\section{QUADRO 5}

PRINCIPAIS ESPAÇOS DE INTERAÇÃO IDENTIFICADOS

\begin{tabular}{|c|c|c|c|}
\hline $\begin{array}{l}\text { ESPAÇOS DE } \\
\text { INTERAÇÃOO }\end{array}$ & AGIVEST & $\begin{array}{l}\text { TECNÓPOLE DO } \\
\text { FUTUROSCOPE }\end{array}$ & $\begin{array}{c}\text { PÓLO DE } \\
\text { INFORMÁTICA }\end{array}$ \\
\hline $\begin{array}{l}\text { Formais face } \\
\text { a face }\end{array}$ & $\begin{array}{l}\text { Cursos e palestras } \\
\text { Planejamento estratégico } \\
\text { Assembléias } \\
\text { Reuniões dentro do segmento }\end{array}$ & $\begin{array}{l}\text { Fórum Universidade e } \\
\text { empresa }\end{array}$ & $\begin{array}{l}\text { Reuniões } \\
\text { Cursos de formação }\end{array}$ \\
\hline $\begin{array}{l}\text { Informais } \\
\text { face a face }\end{array}$ & $\begin{array}{l}\text { Visitas e reuniões nas indústrias } \\
\text { Viagens e visitas a feiras } \\
\text { Confraternizações } \\
\text { Reuniões informais }\end{array}$ & $\begin{array}{l}\text { Café da manhã } \\
\text { Sala de café }\end{array}$ & $\begin{array}{l}\text { Restaurante do Pólo } \\
\text { Sala de café } \\
\text { Confraternizações } \\
\text { eventuais }\end{array}$ \\
\hline
\end{tabular}


No caso do Pólo de Informática e da Tecnópole, a limitada existência e freqüência de espaços que possibilitem a interação entre as pessoas tem sido apontada como uma das causas dos problemas enfrentados na falta de um ambiente de complementaridade de conhecimentos. Esses resultados podem ratificar as evidências de Nonaka e Nishiguchi (200I) ao salientarem que a maior parte do conhecimento, senão todo ele, não é criada por um único indivíduo, mas sim por um processo interativo de experimentação e de diálogo envolvendo vários indivíduos, grupos e organizações.

Esses resultados apontam para os "espaços de interação" como um quinto elemento, não suficientemente identificado na revisão da literatura, mas que influencia fortemente a conectividade e, por conseguinte, a dinâmica de complementaridade de conhecimentos no contexto de uma rede. Tal evidência sugere uma quinta proposição ao esquema conceitual:

Proposição 5 - Os espaços de interação existentes em uma rede influenciam diretamente na conectividade entre os atores e indiretamente na dinâmica de complementaridade de conhecimento.

Esses cinco elementos (pressões contingenciais, coerência, conectividade, mecanismos de coordenação e espaços de interação) foram identificados como fortemente relacionados à dinâmica de complementaridade de conhecimentos no contexto dos casos estudados. No entanto, os resultados sinalizam para outros elementos que podem estar influenciando a complementaridade de conhecimentos e merecem destaque:

a) Aspectos culturais - A dimensão cultural tem sido objeto de amplos interesses no estudo sobre inter-relações organizacionais. Por exemplo, as variáveis culturais como individualismo e coletivismo, estudados por Hofstede (I984), podem estar diretamente relacionadas aos níveis de confiança e de cooperação nos casos pesquisados. Dessa forma, à luz de Hofstede (i984), o executivo brasileiro - mais coletivista - poderá apresentar uma propensão maior para a cooperação, possivelmente, para a socialização de seus conhecimentos, em relação ao executivo francês - mais individualista.

b) Desenvolvimento tecnológico das empresas - A pesquisa, por ter sido conduzida em dois tipos de contextos tecnológicos, um emergente (segmento de internet e multimídia) e outro maduro (segmento de confecções), pode ter tido algum tipo de influência sobre a resistência enfrentada pelos empresários de contextos tecnológicos emergentes em socializarem seus conhecimentos de nível "estratégico". Essa evidência também poderá auxiliar na explicação do fato de as empresas pesquisadas no segmento de confecções apresentarem melhor performance na dinâmica de complementaridade de conhecimentos. 
Pelo fato de esses dois elementos não aparecerem claramente relacionados com a dinâmica de complementaridade de conhecimentos e não suficientemente explorados no campo de estudos, eles irão constar no esquema conceitual somente para sinalizar sua possível influência e sugerir que sejam aprofundados em pesquisas futuras.

\subsection{APRESENTAÇÃO DO ESQUEMA CONCEITUAL}

As cinco proposições apresentadas, elaboradas por meio das evidências empíricas da pesquisa, possibilitaram a construção de um esquema conceitual (Figura 2) que tem por finalidade demonstrar a relação dos principais elementos críticos envolvidos na dinâmica de complementaridade de conhecimentos em um contexto em rede.

\section{FIGURA 2}

ESQUEMA CONCEITUAL DA DINÂMICA DE COMPLEMENTARIDADE DE CONHECIMENTOS

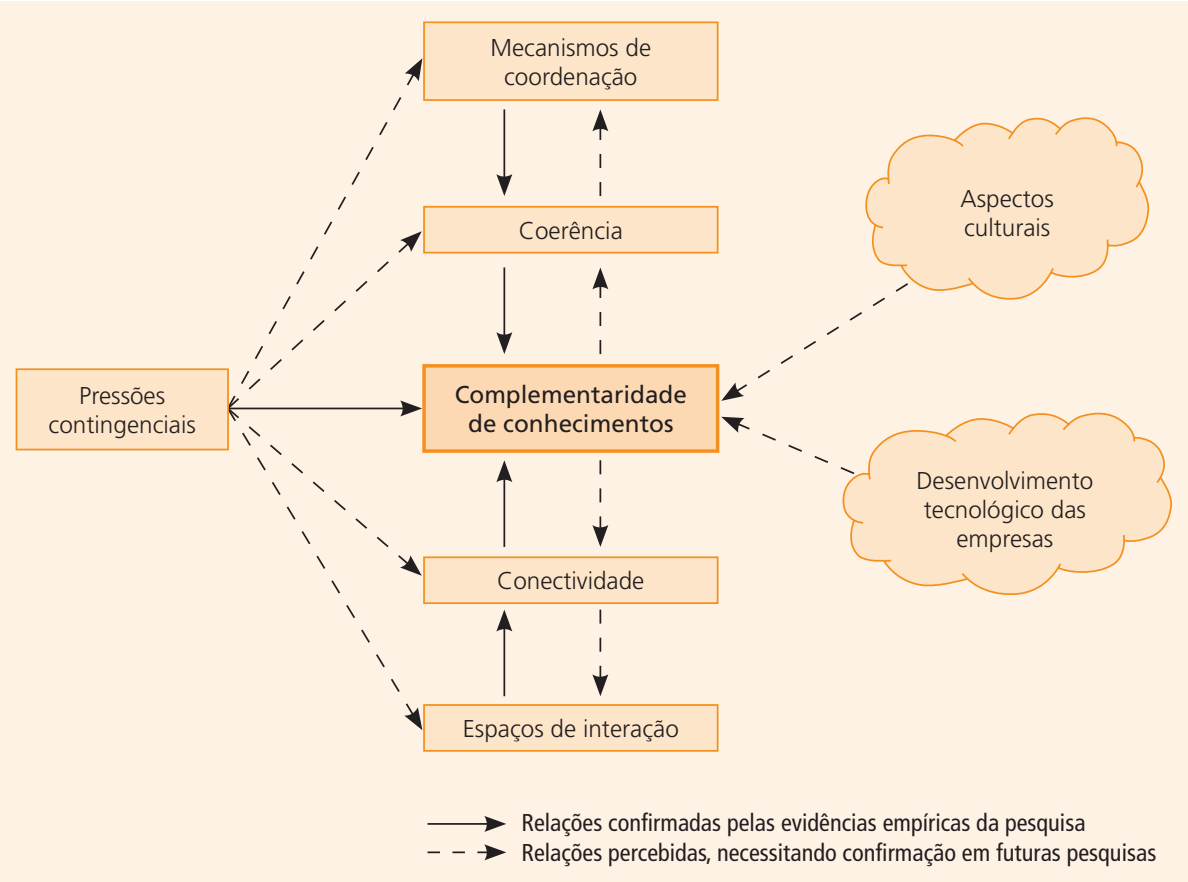

Esse esquema, sem nenhuma pretensão determinista, também poderá auxiliar no entendimento das principais conclusões da pesquisa, a seguir apresentadas: a) As pressões contingenciais, sobretudo de necessidade e de reciprocidade, 
exercem elevada influência para a complementaridade de conhecimentos entre os atores em um contexto em rede; b) A coerência, ou seja, a existência de objetivos comuns entre os atores é condição fundamental para a existência de complementaridade de conhecimentos em um contexto em rede; c) A conectividade, ou seja, o nível de interação entre os atores é essencial para que os elos se comuniquem e possibilitem que o conhecimento seja complementado em uma rede; d) Os mecanismos de coordenação utilizados para facilitar a governança apresentam um papel vital para preservar os interesses comuns (coerência) e, assim, possibilitar a complementaridade de conhecimentos; e) A existência de espaços de interação no contexto de uma rede funcionará como uma plataforma para que a conectividade e a interação sejam estabelecidas, criando, assim, as condições favoráveis para a complementaridade de conhecimentos.

As conclusões delineadas também pretendem contribuir para uma abordagem mais abrangente do conhecimento organizacional, sugerindo a necessidade de deslocar-se de um processo endógeno da firma individual para um processo exógeno, multidirecional e em rede. Também indicam que, para a existência de uma efetiva dinâmica de complementaridade de conhecimentos entre empresas, se deve considerar a natureza tácita, complexa, interdependente e contextual dos conhecimentos relevantes para os processos organizacionais. Essa abordagem vai além do pragmatismo tradicional do knowledge management que tem tratado o conhecimento como um bem de propriedade da empresa e que pode ser facilmente adquirido, estocado e comunicado por meio de TIC entre indivíduos, organizações ou redes.

\section{CONSIDERAÇÕES FINAIS}

De maneira geral, os resultados da pesquisa sinalizam que não é suficiente colocar as empresas juntas para que elas comecem a cooperar e a complementar seus conhecimentos em prol de um ambiente de sinergia e inovação. Essas conclusões põem em evidência várias políticas públicas que buscam o desenvolvimento de regiões por meio do investimento de vultuosos recursos para construir tecnópoles, pólos industriais e outras configurações em rede, sem dar a devida importância a muitas dos elementos críticos identificados no esquema conceitual apresentado.

Os problemas apontados pela presente pesquisa no funcionamento da Tecnópole do Futuroscope e do Pólo de Informática não diminuem a importância e a contribuição desses arranjos. As críticas e teorizações apresentadas objetivam orientar a estruturação e a coordenação do funcionamento desses arranjos para que cada vez mais possam contribuir para o desenvolvimento de PME. Um 
dos principais problemas encontrados na dinâmica de complementaridade de conhecimentos desses casos está relacionado à fragilidade dos mecanismos de coordenação que possibilitem o desenvolvimento de projetos de interesse comum entre os diversos atores.

Outro elemento-chave que merece maior atenção são os espaços de interação que possibilitem o encontro face a face dos empresários, permitindo a socialização de idéias, do "tácito" e do "acaso", fundamentais para o surgimento de um ambiente de inovação. Enfim, para que os "efeitos" de sinergia e de complementaridade aconteçam, é necessário criar um ambiente que possibilite que a confiança e a cooperação sejam no mínimo superiores ao oportunismo e à competição habitualmente presentes nas relações econômicas tradicionais.

Para a área organizacional e nas atividades de gestão, as evidências da pesquisa poderão ser úteis no momento de estruturar, de gerir ou de melhor entender a dinâmica e o funcionamento das empresas em um contexto em rede. Mais precisamente em relação aos casos pesquisados, sugerem-se as seguintes providências: a) a Tecnópole e o Pólo de Informática apresentam a necessidade urgente de um profissional com visão de mercado, conhecimento acadêmico e respaldo das empresas para exercer a atividade de "articulador" ou "animador", no sentido de prospectar e desenvolver projetos de interesses comuns entre as empresas; b) nos três casos pesquisados, recomenda-se a realização de uma cartografia das necessidades e das competências existentes nas empresas e demais instituições presentes para facilitar o mútuo conhecimento e viabilizar projetos de cooperação; c) nos casos da Tecnópole e do Pólo de Informática, é preciso intensificar a realização de atividades que possibilitem a existência de espaços de interação entre os empresários e demais atores.

Destacam-se, também, algumas limitações inerentes à natureza da pesquisa. Ao mesmo tempo que o estudo possibilitou a verticalização da problemática, também causou limitações à generalização dos resultados. Por sua vez, a riqueza de evidências proporcionada pela diversidade dos casos estudados no Brasil e na França também se tornou um fator de complexidade, sobretudo na dificuldade de mensurar os impactos dos elementos de cultura nacional pertinentes aos países estudados. Como sugestão de pesquisas futuras, salienta-se a necessidade de estudos para a compreensão das causas dos diferentes níveis de complementaridade de conhecimentos, sobretudo relacionados aos elementos da cultura nacional e da hostilidade ambiental. Outra sugestão de pesquisa é, com base nos elementos do esquema conceitual, desenvolver pesquisas junto a outras configurações de redes interorganizacionais, com o objetivo de validar ou de criticar os resultados aqui apresentados. 


\section{REFERÊTCIAS}

AMATO NETO, J. Redes de cooperação produtiva e clusters regionais: oportunidades para as pequenas e médias empresas. São Paulo: Atlas, 2000.

BALESTRIN, A.; VARGAS, L. M. A dimensão estratégica das redes horizontais de PME: teorizações e evidências. Revista de Administração Contemporânea (RAC), v. 8, Edição especial, p. 203-228, 2004 .

BOSS, R.W. Trust and managerial problem solving revisited. Group and Organizational Studies, p. 33I-342, Sept. I978.

BROWN, J. E.; HENDRY, C. Industrial districts and supply chains as vehicles for managerial and organizational learning. Studies of Management e Organization, v. 27, n. 4, p. I27-157, I998.

CAGLIO, A. Networks and information technology: Competing through extranets. In: III CEMS - Community of European Management Schools. Louvain-la-Neuve, May 7-9, I998.

CASAROTTO FILHO, N.; PIRES, L. H. Redes de pequenas e médias empresas e desenvolvimento local. São Paulo: Atlas, I999.

CASSON, M.; COX, H. An economic model of inter-firm networks. In: EBERS, M. The formation of inter-organizational networks. Oxford: Oxford University Press, I997.

CASTELLS, M. A era da informação: economia, sociedade e cultura. São Paulo: Paz e Terra, I999. CIBORRA, C.; PATRIOTTA, G. Groupware and teamwork in new product development. In: CIBORRA, C. (Ed.). Groupware and teamwork. Chichester: Wiley, I996.

DIMAGGIO, P.; POWELL, W. The iron cage revisited: institutional isomorphism and collective rationality in organizational fields. American Sociological Review, v. 48, p. I47-I60, I983.

EBERS, M.; JARILLO, J. C. The construction, forms, and consequences of industry networks. International Studies of Management e Organization, v. 27, p. 3-2I, Winter I998.

FLORÉN, H.; TELL, J. The emergent prerequisites of managerial learning in small firm networks. Leadership \& Organization Development Journal, v. 25, n. 3/4, p. 292-307, 2004.

GAMBETTA, D. Can we trust trust? In: __. (Ed.). Trust: making and breaking cooperative relations. Electronic edition. Oxford: University of Oxford, 2000. Chapter I3, p. 213-37. Disponível em: <http://www.sociology.ox.ac.uk/papers/gambetta2i3-237.pdf>.

GRANDORI, A.; SODA, G. Inter-firm networks: Antecedents, mechanisms and forms. Organization Studies, Berlin, v. I6, I995.

GRANT, R. M. Toward a knowledge-based theory of the firm. Strategic Management Journal, v. I7, p. IO9-I22, I996.

HAGE, J.; HOLLINGSWORTH, R. A strategy for the analysis of idea innovation networks and institutions. Organization Studies, v. 2I, p. 97I-I004, 2000.

HOFSTEDE, G. Culture's consequences: international differences in work-related values. London: Sage Publications, I984.

HUMAN, S. E.; PROVAN, K. G. An emergent theory of structure and outcomes in small-firm strategic manufacturing network. Academy of Management Journal, v. 40, n. 2, p. 368-403, 1997.

JARILLO, J. C. On strategic networks. Strategic Management Journal, v. 9, p. 3I-4I, I988.

KOGUT, B.; ZANDER, U. Knowledge of the firm, combinative capabilities, and the replication of technology. Organization Science, v. 3, p. 383-397, i992.

MARCON, M.; MOINET, N. La stratégie-réseau. Paris: Zéro Heure, 2000. 
MILES, R.; SNOW, C. C. Networks organization: new concepts for new forms. California Management Review, v. 28, p. 62-75, I986.

MOWERY, D. C.; ROSENBERG, N. Technology and the pursuit of economic growth. Cambridge: Cambridge University Press, I989.

NOHRIA, N.; ECCLES, R. Networks and organizations: structure, form and action. Boston: Harvard Business School Press, I992.

NONAKA, I. E.; NISHIGUCHI, T. Knowledge emergence. New York: Oxford, 200I.

OLIVER, C. Determinants of interorganizational relationships: integration and future directions. Academy of Management Review, v. I5, n. 2, p. 24I-265, I990.

OLIVER, A. L.; EBERS, M. Networking network studies: an analysis of conceptual configurations in the study of inter-organizational relationships. Organization Studies, v. 19, p. 549-583, I998.

PERROW, C. Small-Firm Networks. In: NOHRIA, N.; ECLES, R. Networks and organizations: structure, form an action. Boston: Harvard Business School Press, I992.

POWELL, W. W. Neither market nor hierarchy. Research in Organizational Behaviour, v. I2, I990. Learning from collaboration: knowledge and networks in the biotechnology and pharmaceutical industries. California Management Review, Berkeley, v. 40, p. 228-240, Spring 1998.

RODAN, S.; GALUNIC, C. More than network structure: how knowledge heterogeneity influences managerial performance and innovativeness. Strategic Management Journal, v. 25, n. 6, p. 54I-562, 2004 .

ROTHWELL, R. Industrial innovation: success, strategy, trends. In: DODGSON, M.; ROTHWELL, R. The handbook of industrial innovation. Cheltenham: Edward Elgar, I995.

SPENCER, J.W. Firms' knowledge-sharing strategies in the global innovation system: empirical evidence from the flat panel display industry. Strategic Management Journal, v. 24, p. 2I7-233, 2003.

SUCHMAN, L. Plans and situated actions: the problem of human-machine communication. New York: Cambridge University Press, 1987.

SYMON, G. Information and communication technologies and network organization: a critical analysis. Journal of Occupational and Organizational Psychology, v. 73, p. 389-4I4, Dec. 2000.

TSAI, W. Social structure of "coopetition" within a multiunit organization: coordination, competition, and intra-organizational knowledge sharing. Organization Science, v. 13, n. 2, p. I79-190, 2002.

VILLWOCK, L. H. Consórcios agroexportadores: estratégia para o desenvolvimento competitivo da cadeia de produção de suínos no RS. Porto Alegre, 200I. Tese (Doutorado) - Programa de PósGraduação em Administração, Universidade Federal do Rio Grande do Sul.

WOLFFENBÜTTEL, A. P. Avaliação do processo de interação universidade-empresa em incubadoras universitárias de empresas: um estudo de caso na incubadora de empresas de base tecnológica da Unisinos. Porto Alegre, 200I. Dissertação (Mestrado) - Programa de Pós-Graduação em Administração, Universidade Federal do Rio Grande do Sul.

\section{TRAMITAÇÃO}

Recebido em 11/7/2006

Aprovado em 1/10/2006 
Copyright of Revista de Administração Mackenzie is the property of Universidade Presbiteriana Mackenzie, RAM-Revista de Administracao Mackenzie and its content may not be copied or emailed to multiple sites or posted to a listserv without the copyright holder's express written permission. However, users may print, download, or email articles for individual use. 